\title{
Ueber die kleinen Schwingungen eines periodisch eingerichteten Systems materieller Punkte.
}

\author{
(Von Herrn E. B. Christoffel in Zürich.)
}

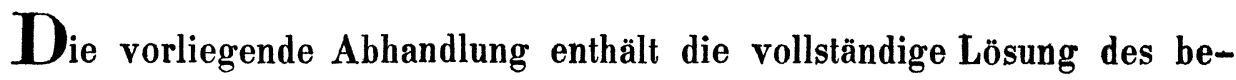
kannten Problems, die unendlich kleinen Schwingungen eines Systems räumlich getrennter materieller Punkte zu bestimmen, zwischen denen anziehende und abstossende Kräfte wirken, und welche in periodisch wiederkehrenden Gruppen im Raume vertheilt sind.

Cauchy, welcher mit diesem Problem der analytischen Optik eine neue Grundlage zu geben gedachte, legte demselben eine grosse Bedeutung bei, wie aus den zahlreichen Abhandlurgen hervorgeht, in denen er sich mit diesem Problem beschäftigt. Dieselben finden sich, soweit sie mir bekannt geworden sind, in den Comptes rendus vom $y^{\text {t... }}$ bis zum $30^{\text {sten }}$ Bande und im $22^{\text {sten }}$ Bande der Memoiren der Pariser Akademie; die ersteren sind zum Theil in den Exercices wiederholt.

Der nächste Weg, welcher sich zur Lösung des vorliegenden Problemes darbietet, besteht in der Ermittlung der Gesetze, nach denen die periodischen Bewegungen des Systems in ebenen Wellen erfolgen. Sind diese bekannt, so liefert der Fouriersche Satz unmittelbar die allgemeinen Formeln für die Bewegung, welche auf einen beliebigen Anfangszustand folgt.

Aber diese Lösung kann nicht als vollendet angesehen werden; im art. III. der folgenden Abhandlung wird nachgewiesen, dass die vielfachen Integrale, auf welche man durch den Fourierschen Satz geführt wird, Elemente enthalten, welche der Lösung vollkommen fremd sind, und welche daher durch eine Reduction jener Integrale ausgeschieden werden müssen.

Durch eine genauere Untersuchung der algebraischen Functionen, welche in diese Integrale eingehen, ist die verlangte Reduction gelungen, zunächst für die einfacheren Ausdrücke, welche Cauchy am Schlusse des \$. 2. seiner Abhandlung über die Farbenzerstreuung giebt, dann auch für die Lösung des allgemeinen Problems.

In der vorliegenden Abhandlung ist der umgekehrte Weg eingeschlagen worden. Nachdem im art. III. nachgewiesen worden ist, welche Re- 


\section{Christoffel, ïber die kleinen Schwingungen eines Systems materieller Punkte.}

ductionen sich bei der Anwendung des Fourierschen Satzes als nothwendig herausstellen, wird die Aufgabe so gestellt und behandelt, dass man von Anfang an die Gewissheit hat, die Lösung in einer Form zu erhalten, welche keiner weitern Reduction mehr bedürftig ist.

Es hat sich auf diesem Wege das merkwürdige Resultat ergeben, dass die Bewegung des zu untersuchenden Systems, auch unter den allgemeinsten Voraussetzungen, stets nur von einer einzigen Klasse analytischer Functionen einer veränderlichen Grösse $t$ abhängt, deren reelle Werthe die seit der anfänglich'en Gleichgewichtsstörung verflossene Zeit bestimmen. Diese Func-

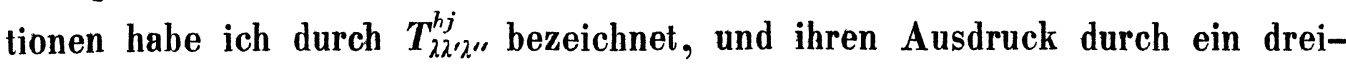
faches Integral mit endlicher Begrenzung im art. IX. Formel 18 angegeben.

I.

Obgleich das Problem, mit welchem die vorliegende Abhandlung sich beschäftigt, allgemein bekannt ist, lässt sich doch eine ausführliche Darlegung desselben nicht füglich umgehen.

Ist $\Pi$ ein System materieller Punkte, so wird dasselbe vollkommen homogen genannt, wenn es folgenden Bedingungen genügt:

1) Alle Punkte des Systems besitzen dieselbe Masse.

2) Sind $a, b, c$ irgend welche Punkte des Systems, und zieht man aus $c$ eine Gerade $c d$ von gleicher Länge und Richtung, wie $a b$, so ist $d$ ebenfalls ein Punkt des Systems.

Fügt man zu diesen Bedingungen das Grundprincip der atomistischen Theorie,

3) dass die Entfernung zweier materieller Punkte, wie klein sie im Uebrigen auch sein mag, doch niemals unter jeder Grenze liegt, so ist das System $I I$ nothwendig ein reticulares, d. h. die materiellen Punkte dieses Systems befinden sich in den Durchschnittspunkten dreier Schaaren von Ebenen; welche den Raum in congruente Parallelepipeda zerlegen *); lässt man den besondern Fall, wo eine oder zwei Kanten dieser Parallepipede unendlich gross sind, unberücksichtigt, so sind also die rechtwinkligen Coordinaten $x, y, z$

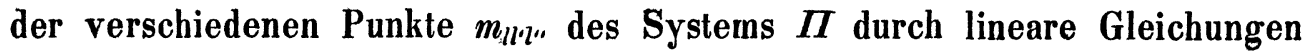

$$
\begin{aligned}
& x=\alpha+a l+a^{\prime} l^{\prime}+a^{\prime \prime} l^{\prime \prime}, \\
& m_{l l^{\prime}{ }^{\prime \prime} .} \quad y=\beta+b l+b^{\prime} l^{\prime}+b^{\prime \prime} l^{\prime \prime}, \\
& z=\gamma+c l+c^{\prime} l^{\prime}+c^{\prime \prime} l^{\prime \prime}
\end{aligned}
$$

*) Man vergleiche Dirichlets Abhandlung über die positiven ternären quadr. Formen, dieses Journal Band 40. 
gegeben, in denen $l, l^{\prime}, l^{\prime \prime}$ jedes ganzzahligen Werthes fähig, und die übrigen Grössen rechter Seite unveränderlich sind.

Diese Definition lässt sich, wie Cauchy bemerkt *), auf homogene Mittel, welche aus chemisch differenten Atomen zusammengesetzt sind, nur anwenden, wenn man sich die Massenpunkte $m_{l l^{\prime \prime}}$ durch Atomengruppen ersetzt denkt, welche um jede Ecke der vorhin erwähnten Parallelepipede in gleicher Weise angeordnet sind.

Wir fügen hiernach zum homogenen System $\Pi$ noch $N$ andere, $\Pi_{1}$, $\Pi_{2}, \ldots \Pi_{N}$, die so geordnet sind, dass die Coordinaten des zu $\Pi_{i}$ gehörigen Punktes $m_{l l \cdot{ }^{\prime \prime}}^{i}$ mit den obigen durch die Gleichungen

$$
m_{l l l \cdots .}^{i} \quad x_{i}-\alpha_{i}=x-\alpha, \quad y_{i}-\beta_{i}=y-\beta, \quad z_{i}-\gamma_{i}=z-\gamma
$$

verbunden sind. $Z$ u jeder Werthengruppe $\left(l, l^{\prime}, l^{\prime \prime}\right)$ gehört dann eine Gruppe materieller Punkte $m_{l \cdot{ }^{\prime \prime}}, m_{l l^{\prime} \imath^{\prime}}^{1}, \ldots m_{l l^{\prime}{ }^{\prime}}^{N}$, die in ihren physikalischen Eigenschaften übereinstimmen oder von einander verschieden sein können. Diese Gruppen wiederholen sich periodisch auf dieselbe Weise, wie die Punkte des Systems $\Pi$; ihre Gesammtheit kann also ebenfalls als ein homogenes System $P$ angesehen werden.

Es sind nun die superponirbaren kleinen Schwingungen eines solchen periodisch eingerichteten Systems $\boldsymbol{P}$, mit denen die gegenwärtige Arbeit sich beschäftigt. Und zwar fügen wir zu den obigen Voraussetzungen über die Einrichtung des Systems $P$ noch die folgenden, welche die in ihm wirkenden Kräfte betreffen.

Wir setzen voraus, dass zwischen den einzelnen materiellen Punkten von $\boldsymbol{P}$ Attractionen (Anziehungen und Abstossungen) wirken, durch welche sie, bei der oben beschriebenen Einrichtung des Systems $\boldsymbol{P}$ im stabilen relativen Gleichgewichte erhalten werden.

Es seien ferner $m_{l l^{\prime \prime}}^{i}$ und $m_{l+n, l^{\prime}+n^{\prime}, l^{\prime \prime}+n^{\prime \prime}}^{k}$ zwei beliebige Punkte von $P$, deren Massen und Coordinaten beziehungsweise $m^{i}, x_{i}, y_{i}, z_{i}$ und $m^{k}, x_{k}^{\prime}, y_{k}^{\prime}$, $z_{k}^{\prime}$ sind. Dann wirkt an $m^{i}$ in Folge der gegenseitigen Attraction beider Punkte in ihrer Verbindungslinie eine Kraft, die bloss von ihrer Entfernung (ik) abhängt, und durch

$$
\frac{\partial[i k]}{\partial(i k)}
$$

bezeichnet werden soll. Die Componenten dieser Kraft werden also ausge-

*) Comptes rendus Tome 9 , pag. 558 . 
drückt durch

$$
\frac{\partial[i k]}{\partial x_{k}^{\prime}}, \frac{\partial[i k]}{\partial y_{k}^{\prime}}, \frac{\partial[i k]}{\partial z_{k}^{\prime}} .
$$

Ueber die Massen $m, m^{\prime}, \ldots m^{N}$, so wie über die Attractionsgesetze, welche durch das Zeichen $\frac{1}{m^{i} m^{k}} \frac{\partial[i k]}{\partial(i k)}$ bei verschiedenen Werthen der Indices $i$ und $k$ dargestellt werden, lassen wir die allgemeinsten Voraussetzungen bestehen; wir setzen also weder zwischen zwei von jenen $N+1$ Massen, noch zwischen zwei von den im Allgemeinen denkbaren $\frac{1}{2}(N+1)(N+2)$ Attractionsgesetzen die Gleichheit voraus.

Da die Differenzen $x_{k}^{\prime}-x_{i}, y_{k}^{\prime}-y_{i}, z_{k}^{\prime}-z_{i}$, von denen die erste nach der oben eingeführten Bezeichnung $=\alpha_{k}-\alpha_{i}+a n+a^{\prime} n^{\prime}+a^{\prime \prime} n^{\prime \prime}$ ist, nur noch von den ganzen Zahlen $n, n^{\prime}, n^{\prime \prime}$ abhängen, welche für den angezogenen Punkt $m_{l l^{\prime} l^{\prime \prime}}^{i}$ die relative Lage des anziehenden Punktes $m_{l+n, l^{\prime}+n^{\prime}, 7^{\prime \prime}+n^{\prime \prime}}^{k}$ bestimmen,

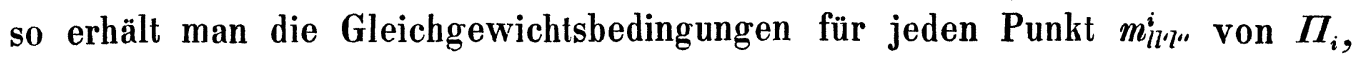
wenn man in den Gleichungen

$$
\mathbf{0}=\sum_{n n^{\prime} n^{\prime \prime}} \sum_{k} \frac{\partial[i k]}{\partial x_{k}^{\prime}}, \quad 0=\sum_{n n^{\prime} n^{\prime \prime}} \sum_{k} \frac{\partial[i k]}{\partial y_{k}^{\prime}}, \quad 0=\sum_{n n^{\prime} n^{\prime \prime}} \sum_{k} \frac{\partial[i k]}{\partial z_{k}^{\prime}}
$$

die erste Summation über alle ganzzahligen Werthe von $n, n^{\prime}, n^{\prime \prime}$, die zweite über die Werthe $k=0,1, \ldots N$ erstreckt, mit Weglassung des einzigen Gliedes $\left(n=n^{\prime}=n^{\prime \prime}=0, k=i\right)$.

Ertheilt man hierauf dem Index $i$ der Reihe nach die Werthe $0,1, \ldots N$, so ergeben sich die vollständigen Bedingungen für das Gleichgewicht des Systems $P$.

II.

Da das relative Gleichgewicht von $P$ der Voraussetzung gemäss stabil ist, so kann man seinen Punkten so kleine relative Ortsänderungen und Geschwindigkeiten ertheilen, dass dieselben in der nachfolgenden Bewegung unaufhörlich innerhalb beliebig enge gewählter Grenzen beharren.

Man kann also bewirken, dass jede relative Ortsänderung fortwährend unendlich klein bleibt. Dann lassen sich die beschleunigenden Kräfte in jedem Augenblicke durch die Ortsänderungen linear ausdrücken. Folglich sind die Bewegungen in unendlich kleinen relativen Ortsänderungen superponirbar.

Bezeichnet man zur Zeit $\boldsymbol{t}$ die zu den Axen parallelen Verschiebungen

$$
\left\{\begin{array}{l}
\text { des Punktes } m_{l l^{\prime \prime} l^{\prime \prime}}^{i} \text { durch } \xi_{l l^{\prime} l^{\prime \prime}}^{i}=\xi_{i}, \eta_{l l^{\prime} l^{\prime \prime}}^{i}=\eta_{i}, \zeta_{l l^{\prime}{ }^{\prime}}^{i}=\zeta_{i}, \\
\text { des Punktes } m_{l+n, l^{\prime}+n^{\prime}, l^{\prime}+n^{\prime \prime}}^{k} \operatorname{durch} \xi_{k}^{\prime}, \eta_{k}^{\prime}, \zeta_{k}^{\prime},
\end{array}\right.
$$




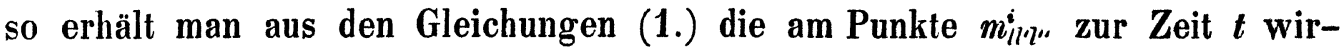
kenden Componenten, wenn man auf der rechten Seite überall und für jedes $k$ $x_{k}^{\prime}-x_{i}, \quad y_{k}^{\prime}-y_{i}, \quad z_{k}^{\prime}-z_{i}$ durch $x_{k}^{\prime}-x_{i}+\xi_{k}^{\prime}-\xi_{i}, y_{k}^{\prime}-y_{i}+\eta_{k}^{\prime}-\eta_{i}, z_{k}^{\prime}-z_{i}+\zeta_{k}^{\prime}-\zeta_{i}$ ersetzt, also $x_{k}^{\prime}, y_{k}^{\prime}, z_{k}^{\prime}$ bezüglich um $\xi_{k}^{\prime}-\xi_{i}, \eta_{k}^{\prime}-\eta_{i}, \zeta_{k}^{\prime}-\zeta_{i}$ wachsen lässt.

Daraus ergeben sich die Gleichungen für die superponirbaren Bewegungen des Systems $\boldsymbol{P}$ in folgender Gestalt:

$$
\left\{\begin{array}{l}
m^{i} \frac{\partial^{2} \xi_{l l^{\prime} \eta^{\prime \prime}}}{\partial t^{2}}=\sum_{n n^{\prime} n^{\prime \prime}} \sum_{k}\left[\frac{\partial^{2}[i k]}{\partial x_{k}^{\prime 2}}\left(\xi_{k}^{\prime}-\xi_{i}\right)+\frac{\partial^{2}[i k]}{\partial x_{k}^{\prime} \partial y_{k}^{\prime}}\left(\eta_{k}^{\prime}-\eta_{i}\right)+\frac{\partial^{2}[i k]}{\partial x_{k}^{\prime} \partial z_{k}^{\prime}}\left(\zeta_{k}-\zeta_{i}\right)\right] \\
m^{i} \frac{\partial^{2} \eta_{l l^{\prime \prime \prime}}^{i}}{\partial t^{2}}=\sum_{n n^{\prime} n^{\prime \prime}} \sum_{k}\left[\frac{\partial^{2}[i k]}{\partial x_{k}^{\prime} \partial y_{k}^{\prime}}\left(\xi_{k}^{\prime}-\xi_{i}\right)+\frac{\partial^{2}[i k]}{\partial y_{k}^{\prime 2}}\left(\eta_{k}^{\prime}-\eta_{i}\right)+\frac{\partial^{2}[i k]}{\partial y_{k}^{\prime} \partial z_{k}^{\prime}}\left(\zeta_{k}^{\prime}-\zeta_{i}\right)\right], \\
m^{i} \frac{\partial^{2} \zeta_{i l^{\prime} l^{\prime \prime}}}{\partial t^{2}}=\sum_{n n^{\prime} u^{\prime \prime}} \sum_{k}\left[\frac{\partial^{2}[i k]}{\partial x_{k}^{\prime} \partial z_{k}^{\prime}}\left(\xi_{k}^{\prime}-\xi_{i}\right)+\frac{\partial^{2}[i k]}{\partial y_{k}^{\prime} \partial z_{k}^{\prime}}\left(\eta_{k}^{\prime}-\eta_{i}\right)+\frac{\partial^{2}[i k]}{\partial z_{k}^{\prime 2}}\left(\zeta_{k}^{\prime}-\zeta_{i}\right)\right]
\end{array}\right.
$$

Dieselben müssen für alle ganzzahligen Werthe von $l, l^{\prime}, l^{\prime \prime}$ und für $i=0,1, \ldots N$ bestehen. Die Bedingung, dass bei den Summationen das Glied $\left(n=n^{\prime}=n^{\prime \prime}=0, k=i\right)$ weggelassen werden soll, braucht jetzt nicht mehr berücksichtigt zu werden, da bei diesen Werthen der Summationsindices die Factoren $\xi_{k}^{\prime}-\xi_{i}$ u. s. w. sich auf Null reduciren.

\section{III:}

Seit den Untersuchungen Cauchys über die reticularen Systeme pflegt man den Anfangszustand eines solchen Systems durch stetige Functionen der Coordinaten auszudrücken, welche nicht bloss den materiellen Punkten des Systems, sondern auch den sie umgebenden massenfreien Punkten Anfangsverschiebungen und Geschwindigkeiten anweisen.

Indem man hierauf die Differentialgleichungen der Bewegung auf alle Punkte des Raumes ausdehnt, bestimmt man mittelst des Fourierschen Satzes eine Bewegung des continuirlichen Raumes, an welcher die in ihn eingetauchten materiellen P.unkte ihrem Orte gemäss theilnehmen.

Bei diesem Verfahren wird die Bewegung des materiellen Punktsystems abhängig gemacht, nicht bloss von seinem eigenen Anfangszustande, was für jeden einzelnen Punkt desselben in irgend einem Umfange der Fall sein muss, sondern auch noch vom Anfangszustande des massenfreien Raumes, was in Wirklichkeit nicht der Fall sein kann. Von diesen, der Lösung nothwendig fremden Elementen kann dieselbe nur durch die Reduction der vielfachen In- 
tegrale befreit werden, welche durch den Fourierschen Satz eingeführt worden sind.

Um diesen Uebelstand zu vermeiden, geben wir den Anfangszustand des Systems $\boldsymbol{P}$ unmittelbar durch die Verschiebungen und Geschwindigkeiten seiner eigenen Punkte. Sei

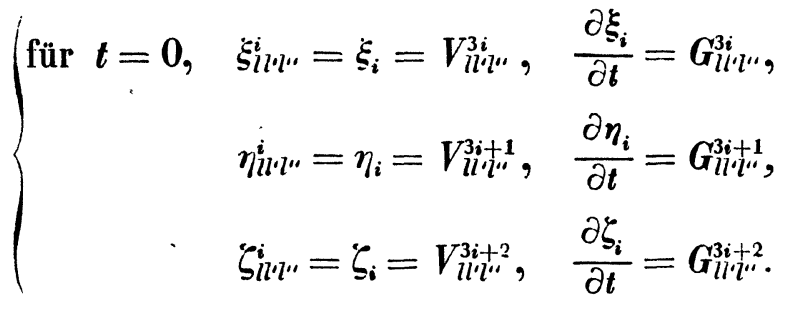

Dann lässt sich das zu lösende Problem wie folgt aussprechen:

Es sollen, für alle ganzzahligen Werthe von $l, l^{\prime}, l^{\prime \prime}$ und für $i=0$, $1, \ldots N$

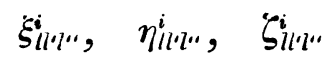

als Functionen der Zeit $t$ und der Anfangswerthe $V$ und $G$ den Gleichungen (3.) und (4.) gemäss bestimmt werden.

IV.

Unsere nächste Aufgabe besteht darin, zu ermitteln, in welcher Weise die gesuchten Functionen von den Anfangswerthen abhängen.

$\mathrm{Zu}$ diesem Zwecke lassen wir an Stelle des durch die Gleichung (4.) dargestellten Anfangszustandes einen andern treten, in welchem nur der Punkt

$$
m_{\lambda+l, \lambda^{\prime}+l^{\prime}, \lambda^{\prime \prime}+l^{\prime \prime}}^{k}
$$

in seinem Gleichgewichte gestört worden ist, und auch diesem Punkte nur unendlich kleine Verschiebungen und Geschwindigkeiten $\partial V, \partial V^{\prime}, \partial V^{\prime \prime}$ und $\partial G, \partial G^{\prime}, \partial G^{\prime \prime}$ ertheilt worden sind. Aus dieser Gleichgewichtsstörung entspringt eine Bewegung des Systems $P$, in welchem wir die Verschiebungen des Punktes

$$
m_{l l \cdot l^{\prime \prime}}
$$

zur Zeit $t$ durch $X, Y, Z$ bezeichnen wollen.

Wir wollen zweitens voraussetzen, dass man zur Zeit $t=0$, ohne des Gleichgewicht der übrigen Punkte des Systems zu stören, die Verschie- 
bungen $\partial V, \partial V^{\prime}, \partial V^{\prime \prime}$ und die Geschwindigkeiten $\partial G, \partial G^{\prime}, \partial G^{\prime \prime}$ dem Punkte $m_{\lambda \lambda^{\prime} \cdot \lambda^{\prime \prime}}^{k}$

ertheilt habe. Bezeichnet man alsdann in der hieraus erfolgenden Bewegung des Systems $P$ die Verschiebungen des Punktes

$$
m_{(x)}^{i}
$$

zur. Zeit $t$ durch $\mathfrak{X}, \mathfrak{Y}, \mathcal{B}$, so muss wegen der Einrichtung des Systems $P$

$$
\boldsymbol{X}=\mathfrak{X}, \quad \boldsymbol{Y}=\mathfrak{Y}, \quad \boldsymbol{Z}=\mathbf{3}
$$

sein. In der That werden die im zweiten Falle gemachten Voraussetzungen mit den auf den ersten Fall bezüglichen völlig identisch, wenn man das System $P$ ohne Drehung so lange verschiebt, bis der Punkt $m_{\lambda \lambda^{\prime} \cdot{ }^{\prime \prime}}$ nach

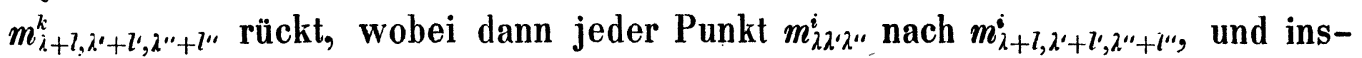
besondere $m_{1 x y)}^{i}$ nach $m_{l l^{\prime \prime \prime}}^{i}$ geschoben wird.

Für die Grössen $X, \mathfrak{X}, Y, \ldots$ lassen sich aber leicht bestimmte Ausdrücke finden. Superponirt man nämlich jeden der beiden Anfangszustände, aus denen sie entspringen, mit dem durch die Gleichungen (4.) bestimmten, so werden im ersten Falle die Anfangswerthe $\left(V^{3 k}, G^{3 k}, V^{3 k+1}, \ldots\right)_{\lambda+l, \lambda^{\prime}+l^{\prime}, \lambda^{\prime \prime}+l^{\prime \prime}}$ um $\partial V, \partial G, \partial V^{\prime}, \ldots$, und in Folge dessen die Verschiebungen $(\xi, \eta, \zeta)_{l i \cdot \cdots}^{i}$ um $X, Y, Z$ vermehrt; im zweiten Falle, wo die Aenderungen $\partial V, \partial G$, $\partial V^{\prime}, \ldots$ den Anfangswerthen $\left(V^{3 k}, G^{3 k}, V^{3 k+1}, \ldots\right)_{\lambda \lambda_{\lambda}{ }^{4}}$ ertheilt werden, ändern $\operatorname{sich}(\xi, \eta, \zeta)_{\text {ixo }}^{i}$ um $\mathfrak{X}, \mathfrak{Y}, 3$.

Drückt man demnach die Verschiebungen $X, \mathfrak{X}, Y, \ldots$ durch die

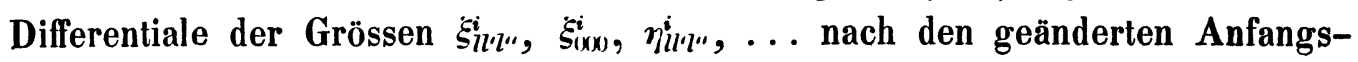
werthen aus, so ergibt sich aus ihrer Gleichheit z. B.

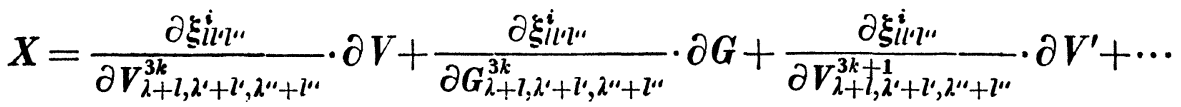

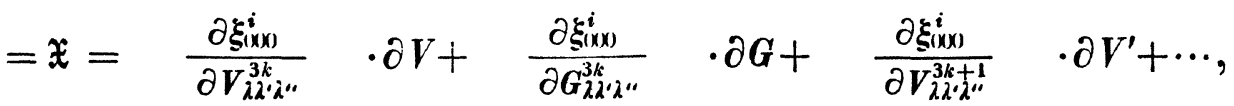

und allgemein, wenn man einen der Buchstaben $\xi, \eta, \zeta$ durch $(\xi, \eta, \zeta)$, und einen der Buchstaben $V, G$ durch $(V, G)$ bezeichnet

$$
\frac{\partial(\xi, \eta, \zeta)_{l l^{\prime \prime \prime}}^{i}}{\partial(\boldsymbol{V}, \boldsymbol{G})_{\lambda+l, \lambda^{\prime}+l^{\prime}, \lambda^{\prime \prime}+l^{\prime \prime}}^{j}}=\frac{\partial(\xi, \eta, \zeta)_{0 \times 0)}^{i}}{\partial(\boldsymbol{V}, \boldsymbol{G})_{\lambda \lambda^{\prime} \cdot \lambda^{\prime \prime}}^{j}},
$$

für $i=0,1, \ldots N, j=0,1, \ldots 3 N+2$, und alle ganzzahligen Werthe von $\lambda, \lambda^{\prime}, \lambda^{\prime \prime}, l, l^{\prime}, l^{\prime \prime}$. 
280 Christoffel, über die kleinen Schwingungen eines Systems materieller Punkte.

Wir setzen von jetzt an

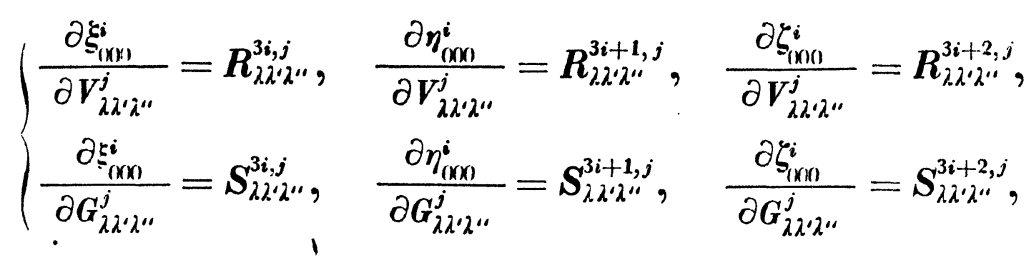

und werden im folgenden art. zeigen, wie sich die Bestimmung der Functionen $\xi, \eta, \zeta$ vollständig auf die Ermittlung der Functionen $\boldsymbol{R}$ und $\boldsymbol{S}$ zurückführen lässt.

V.

Zunächst ergiebt sich aus den für $t=0$ stattfindenden Gleichungen $\xi_{(\times)}^{i}=V_{(x)}^{3 i}, \frac{\partial \xi_{(x)}^{i}}{\partial t}=G_{(x \times)}^{3 i}, \eta_{(\times)}^{i}=V_{0 \times(x)}^{3 i+1}$, u. s. w. mit Hülfe der Gleichungen (b.), dass für $t=0$, mit einziger Ausnahme von

$$
R_{(x \times)}^{j, j} \quad \text { und } \frac{\partial S_{0(x)}^{j, j}}{\partial t} \quad(j=0,1, \ldots 3 N+2),
$$

welche gleich 1 werden, alle übrigen Functionen $R, S, \frac{\partial R}{\partial t}, \frac{\partial S}{\partial t}$ verschwinden.

Ferner erhält man aus den Gleichungen (3.), indem man sie nach einem Anfangswerthe differentiirt, mit Hülfe der Gleichungen (5.) Differentialgleichungen, denen die Functionen $\boldsymbol{R}$ und $\boldsymbol{S}$ genügen müssen.

Beachtet man, dass nach (5.) und (6.) auf Grund der in art. II. eingeführten Bezeichnung

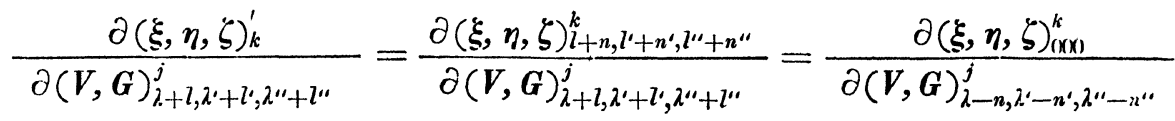

$$
\begin{aligned}
& =\left(\boldsymbol{P}^{3 k, j}, \boldsymbol{P}^{3 k+1, j}, \boldsymbol{P}^{3 k+2, j}\right)_{\lambda-n, \lambda^{\prime}-n^{\prime}, \hat{i}^{\prime \prime}-n^{\prime \prime}}, \\
& \frac{\partial(\xi, \eta, \zeta)_{i}}{\partial(V, G)_{\lambda+l, \lambda^{\prime}+l^{\prime}, \lambda^{\prime \prime}+l^{\prime \prime}}^{j}}=\left(P^{3 i, j}, P^{3 i+1, j}, P^{3 i+2, j}\right)_{\lambda \lambda^{\prime} \lambda^{\prime \prime}}
\end{aligned}
$$

ist, wo $\boldsymbol{P}$ durch $\boldsymbol{R}$ oder $\boldsymbol{S}$ zu ersetzen ist, jenachdem man nach $\boldsymbol{V}$ oder $\boldsymbol{G}$ differentiirt, so erkennt man sofort, dass man, um die Gleichungen (3.) nach $(V, G)_{\lambda+l, \lambda^{\prime}+l^{\prime}, \lambda^{\prime \prime}+l^{\prime \prime}}^{j} \mathrm{zu}$ differentiiren, nur nöthig hat, dort überall

$$
\xi_{i}, \eta_{i}, \zeta_{i} \text { durch } P_{\lambda \lambda^{\prime} \lambda^{\prime \prime}}^{3 i, j}, P_{\lambda \lambda^{\prime} \cdot \lambda^{\prime \prime}}^{3 i+1, j}, P_{\lambda \lambda^{\prime} \lambda^{\prime \prime}}^{3 i+2, j}
$$


und die Differenzen

durch

$$
\xi_{k}^{\prime}-\xi_{i}, \quad \eta_{k}^{\prime}-\eta_{i}, \quad \zeta_{k}^{\prime}-\zeta_{i}
$$

$$
\begin{array}{ccc}
\boldsymbol{P}_{\lambda-n, \lambda^{\prime}-n^{\prime}, \lambda^{\prime \prime}-n^{\prime \prime}}^{3 k, j}-\boldsymbol{P}_{\lambda \lambda^{\prime} \lambda^{\prime \prime}}^{3 i, j} & \boldsymbol{P}_{\lambda-n, \lambda^{\prime}-n^{\prime}, \lambda^{\prime \prime}-n^{\prime \prime}}^{3 k+1, P_{\lambda \lambda^{\prime} \lambda^{\prime \prime}}^{3 i+1, j}} & \boldsymbol{P}_{\lambda-n, \lambda^{\prime}-n^{\prime}, \lambda^{\prime \prime}-n^{\prime \prime}}^{3 k+2, P_{2}}-\boldsymbol{P}_{\lambda \lambda^{\prime} \lambda^{\prime \prime}}^{3 i+2, j} \\
=\mathfrak{P}^{3 k, 3 i} & =\mathfrak{P}^{3 k+1,3 i+1} & =\mathfrak{P}^{3 k+2,3 i+2}
\end{array}
$$

zu ersetzen.

Behält man diese Bezeichnung zur Abkürzung bei, so ergiebt sich also

(7.)

$$
\left\{\begin{array}{l}
m^{i} \frac{\partial^{2} P_{\lambda \lambda^{\prime} \cdot \lambda^{\prime \prime}}^{3 i, j}}{\partial t^{2}}=\sum_{n n^{\prime} n^{\prime \prime}} \sum_{k}\left[\frac{\partial^{2}[i k]}{\partial x_{k}^{\prime 2}} \mathfrak{P}^{3 k, 3 i}+\frac{\partial^{2}[i k]}{\partial x_{k}^{\prime} \partial y_{k}^{\prime}} \mathfrak{P}^{3 k+1,3 i+1}+\frac{\partial^{2}[i k]}{\partial x_{k}^{\prime} \partial z_{k}^{\prime}} \mathfrak{P}^{3 k+2,3 i+2}\right], \\
m^{i} \frac{\partial^{2} P_{\lambda \lambda^{\prime \prime} \cdot \lambda^{\prime \prime}}^{3, j}}{\partial t^{2}}=\sum_{n n^{\prime} n^{\prime \prime}} \sum_{k}\left[\frac{\partial^{2}[i k]}{\partial x_{k}^{\prime} \partial y_{k}^{\prime}} \mathfrak{P}^{3 k, 3 i}+\frac{\partial^{2}[i k]}{\partial y_{k}^{\prime 2}} \mathfrak{P}^{3 k+1,3 i+1}+\frac{\partial^{2}[i k]}{\partial y_{k}^{\prime} \partial z_{k}^{\prime}} \mathfrak{P}^{3 k+2,3 i+2}\right], \\
m^{i} \frac{\partial^{2} P_{\lambda \lambda^{\prime} \lambda^{\prime \prime}}^{3 i+2, j}}{\partial t^{2}}=\sum_{n n^{\prime} n^{\prime \prime}} \sum_{k}\left[\frac{\partial^{2}[i k]}{\partial x_{k}^{\prime} \partial z_{k}^{\prime}} \mathfrak{P}^{3 k, 3 i}+\frac{\partial^{2}[i k]}{\partial y_{k}^{\prime} \partial z_{k}^{\prime}} \mathfrak{P}^{3 k+1,3 i+1}+\frac{\partial^{2}[i k]}{\partial z_{k}^{\prime 2}} \mathfrak{P}^{3 k+2,3 i+2}\right] .
\end{array}\right.
$$

Fügt man zu diesen Gleichungen die Bedingung, dass

für $t=0$ und $j=0,1, \ldots 3 N+2$

$$
\text { (8.) } \quad \boldsymbol{P}_{0 \times 0}^{j, j}=\mathfrak{A}^{j}, \quad \frac{\partial P_{0 \times 0}^{j, j}}{\partial t}=\mathfrak{B}^{j}
$$

werde, und alle übrigen Functionen $P$ und $\frac{\partial P}{\partial t}$ verschwinden,

so wird offenbar

I. $P=R$, wenn man alle $\mathfrak{A}^{j}=1$, alle $\mathfrak{B}^{j}=0$ setzt;

II. $P=S$, wenn man alle $\mathfrak{A}^{j}=0$, alle $\mathfrak{B}^{j}=1$ setzt, gleiche Indices bei $P, R$ und $S$ vorausgesetzt.

Diese Bedingungen zeigen, was auch für sich klar ist, dass die Functionen $R$ und $S$ von den Anfangswerthen $V$ und $G$ unabhängig sind; also sind (5. und 6.) die Verschiebungen $\xi, \eta$ und $\zeta$ lineare Functionen derselben. Da diese Functionen verschwinden, wenn man alle Anfangswerthe auf Null reducirt, so erhält man aus den Gleichungen (5.) und (6.)

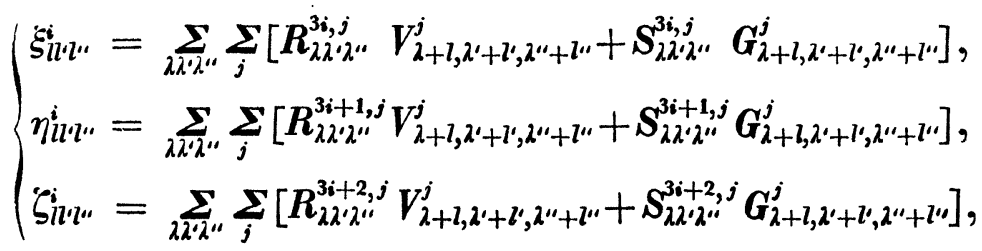


wo von den beiden Summationen sich die erste über alle ganzzahligen Werthe von $\lambda, \lambda^{\prime}, \lambda^{\prime \prime}$, und die zweite sich über die Werthe $j=0,1,2, \ldots 3 N+2$ erstreckt.

VI.

Man kann eine Particularlösung der Gleichungen (7.) finden, indem man jede Function $\boldsymbol{P}_{l l^{\prime} u^{\prime \prime}}$ zur Exponentialgrösse $e^{\left(l u+l^{\prime} u^{\prime}+l^{\prime \prime} u^{\prime \prime}\right) \gamma-1}$ proportional setzt, wobei $u, u^{\prime}, u^{\prime \prime}$ Constanten bedeuten, welche willkürlich bleiben. Durch Superposition solcher Particularlösungen muss man hierauf den Anfangsbedingungen (8.) Genüge leisten.

In Folge der besondern Beschaffenheit dieser Anfangsbedingungen ist es leicht, im Voraus das Resultat der eben bezeichneten Superposition zu erkennen. Es sei $\Omega$ eine Function der Variabeln $t, u, u^{\prime}$, $u^{\prime \prime}$, welche ebenso wie ihre erste Derivirte $\frac{\partial \Omega}{\partial t}$ für $t=0$ von $u, u^{\prime}, u^{\prime \prime}$ unabhängig wird. Entwickelt man diese beiden Functionen für alle zwischen $-\pi$ und $\pi$ enthaltenen Werthe von $u, u^{\prime}$, $u^{\prime \prime}$ nach Exponentialgrössen $e^{\left(\lambda u+\lambda^{\prime} u^{\prime}+\lambda^{\prime \prime} u^{\prime \prime}\right) V^{\prime}-1}$, so verschwinden für $t=0$ alle Coefficienten in diesen Entwicklungen bis auf diejenigen, welche der Voraussetzung $\lambda=\lambda^{\prime}=\lambda^{\prime \prime}=0$ entsprechen. Dies ist genau die von den Functionen $\boldsymbol{P}_{\lambda \lambda^{\prime}{ }^{\prime \prime}}$ verlangte Eigenschaft. Wir setzen demnach

$$
\text { (10.) } \quad P_{\lambda^{\prime} \lambda^{\prime \prime \prime}}^{h, j}=\frac{1}{(2 \pi)^{3}} \int \Omega^{h j} e^{\left(\lambda u+\lambda^{\prime} u^{\prime}+\lambda^{\prime \prime} u^{\prime \prime}\right) v-1} \partial u \partial u^{\prime} \partial u^{\prime \prime} \text {, }
$$

wo jede Integration zwischen den Grenzen $-\pi$ und $\pi$ auszuführen ist.

Dann verlangen die Anfangsbedingungen (8.), dass man für $t=0$ habe

$$
\text { (11.) } \quad \Omega^{j j}=\mathfrak{A}^{j}, \quad \frac{\partial \Omega^{j j}}{\partial t}=\mathfrak{B}^{j}, \quad \Omega^{h j}=0, \quad \frac{\partial \Omega^{h j}}{\partial t}=0,
$$

wenn $h$ von $j$ verschieden ist.

Ferner ergiebt sich aus (10.)

$$
P_{\lambda-n, \lambda^{\prime}-n^{\prime}, \lambda^{\prime \prime}-n^{\prime \prime}}^{h j}=\frac{1}{(2 \pi)^{3}} \int \Omega^{h j} e^{(\lambda u+\ldots) v-1} \cdot e^{-(n u+\ldots) v-1} \partial u \partial u^{\prime} \partial u^{\prime \prime} .
$$

Wir setzen diese Ausdrücke in die Gleichungen (7.) ein, und bedienen uns folgender abkürzenden Bezeichnung:

$$
e^{-\left(n u+n^{\prime} \omega^{\prime}+n^{\prime \prime} u^{\prime \prime}\right) v-1}=\omega,
$$


(ferner in der ersten Gleichung:

$$
\begin{array}{ll}
\sum_{n n^{\prime} n^{\prime \prime}} \sum_{k=0}^{k=N} \frac{\partial^{2}[i k]}{\partial x_{k}^{\prime 2}}-\sum_{n n^{\prime} n^{\prime \prime}} \frac{\partial^{2}[i i]}{\partial x_{i}^{\prime 2}} \cdot \omega=(3 i, 3 i), & -\sum_{n n^{\prime} n^{\prime \prime}} \frac{\partial^{\prime}[i k]}{\partial x_{k}^{\prime 2}} \cdot \omega=(3 i, 3 k), \\
\sum \sum \frac{\partial^{2}[i k]}{\partial x_{k}^{\prime} \partial y_{k}^{\prime}}-\sum \frac{\partial^{2}[i i]}{\partial x_{i}^{\prime} \partial y_{i}^{\prime}} \cdot \omega=(3 i, 3 i+1), & -\sum \frac{\partial^{2}[i k]}{\partial x_{k}^{\prime} \partial y_{k}^{\prime}} \cdot \omega=(3 i, 3 k+1), \\
\Sigma \sum \frac{\partial^{2}[i k]}{\partial x_{k}^{\prime} \partial z_{k}^{\prime}}-\sum \frac{\partial^{2}[i i]}{\partial x_{i}^{\prime} \partial z_{i}^{\prime}} \cdot \omega=(3 i, 3 i+2), & -\sum \frac{\partial^{s}[i k]}{\partial x_{k}^{\prime} \partial z_{k}^{\prime}} \cdot \omega=(3 i, 3 k+2),
\end{array}
$$

in der zweiten

$$
\begin{aligned}
& \Sigma \Sigma \frac{\partial^{2}[i k]}{\partial x_{k}^{\prime} \partial y_{k}^{\prime}}-\sum \frac{\partial^{2}[i i]}{\partial x_{i}^{\prime} \partial y_{i}^{\prime}} \cdot \omega=(3 i+1,3 i), \quad-\Sigma \frac{\partial^{2}[i k]}{\partial x_{k}^{\prime} \partial y_{k}^{\prime}} \cdot \omega=(3 i+1,3 k), \\
& \Sigma \Sigma \frac{\partial^{2}[i k]}{\partial y_{k}^{\prime 2}}-\Sigma \frac{\partial^{2}[i i]}{\partial y_{i}^{\prime 2}} \cdot \omega=(3 i+1,3 i+1),-\Sigma \frac{\partial^{2}[i k]}{\partial y_{k}^{\prime 2}} \cdot \omega=(3 i+1,3 k+1), \\
& \Sigma \Sigma \frac{\partial^{2}[i k]}{\partial y_{k}^{\prime} \partial z_{k}^{\prime}}-\Sigma \frac{\partial^{2}[i i]}{\partial y_{i}^{\prime} \partial z_{i}^{\prime}} \cdot \omega=(3 i+1,3 i+2), \quad-\Sigma \frac{\partial^{2}[i k]}{\partial y_{k}^{\prime} \partial z_{k}^{\prime}} \cdot \omega=(3 i+1,3 k+2), \\
& \Sigma \Sigma \frac{\partial^{2}[i k]}{\partial x_{k}^{\prime} \partial z_{k}^{\prime}}-\Sigma \frac{\partial^{2}[i i]}{\partial x_{i}^{\prime} \partial z_{i}^{\prime}} \cdot \omega=(3 i+2,3 i), \quad-\Sigma \frac{\partial^{2}[i k]}{\partial x_{k}^{\prime} \partial z_{k}^{\prime}} \cdot \omega=(3 i+2,3 k), \\
& \Sigma \Sigma \frac{\partial^{2}[i k]}{\partial y_{k}^{\prime} \partial z_{k}^{\prime}}-\Sigma \frac{\partial^{2}[i i]}{\partial y_{i}^{\prime} \partial z_{i}^{\prime}} \cdot \omega=(3 i+2,3 i+1), \quad-\Sigma \frac{\partial^{2}[i k]}{\partial y_{k}^{\prime} \partial z_{k}^{\prime}} \cdot \omega=(3 i+2,3 k+1), \\
& \Sigma \Sigma \frac{\partial^{2}[i k]}{\partial z_{k}^{\prime 2}}-\Sigma \frac{\partial^{2}[i i]}{\partial z_{i}^{\prime 2}} \cdot \omega=(3 i+2,3 i+2), \quad-\Sigma \frac{\partial^{2}[i k]}{\partial z_{k}^{\prime 2}} \cdot \omega=(3 i+2,3 k+2),
\end{aligned}
$$

wo die Gleichungen der zweiten Verticalreihe $k$ und $i$ als von einander verschieden voraussetzen.

Unterscheidet man in den Gleichungen (7.) die beiden Fälle, wo $k=i$ oder $k$ von $i$ verschieden ist, so erkennt man, dass dieselben sich mit Hülfe der vorstehenden Bezeichnungen in die folgenden verwandeln, in denen

$$
\frac{1}{(2 \pi)^{3}} e^{\left(\lambda u+\lambda^{\prime} u^{\prime}+\lambda^{\prime \prime} u^{\prime \prime}\right) v-1} d u d u^{\prime} d u^{\prime \prime}=\partial E_{\lambda \lambda^{\prime} \cdot \lambda^{\prime \prime}}
$$

gesetzt ist:

$$
\begin{aligned}
& 0=\int \partial E^{\lambda \lambda^{\prime} \cdot{ }^{\prime \prime}}\left[m^{i} \frac{\partial^{2} \Omega^{3 i, j}}{\partial t^{2}}+\sum_{k}\left((3 i, 3 k) \Omega^{3 k, j}+(3 i, 3 k+1) \Omega^{3 k+1, j}+(3 i, 3 k+2) \Omega^{3 k+2, j}\right)\right], \\
& 0=\int \partial E_{\lambda \lambda^{\prime \prime} \lambda^{\prime \prime}}\left[m^{i} \frac{\partial^{2} \Omega^{3 i+1, j}}{\partial t^{2}}+\sum_{k}\left((3 i+1,3 k) \Omega^{3 k, j}+(3 i+1,3 k+1) \Omega^{3 k+1, j}+(3 i+1,3 k+2) \Omega^{3 k+2, j}\right)\right], \\
& 0=\int \partial E_{\lambda \lambda^{\prime \prime}{ }^{\prime \prime}}\left[m^{i} \frac{\partial^{2} \Omega^{3 i+2, j}}{\partial t^{2}}+\sum_{k}\left((3 i+2,3 k) \Omega^{3 k, j}+(3 i+2,3 k+1) \Omega^{3 k+1, j}+(3 i+2,3 k+2) \Omega^{3 k+2, j}\right)\right],
\end{aligned}
$$

wo $k$ bei der Summation die Werthe $0,1, \ldots N$ durchläuft. 
284 Christoffel, über die kleinen Schwingungen eines Systems materieller Punkte.

Diese Gleichungen müssen für alle ganzzahligen Werthe von $\lambda, \lambda^{\prime}, \lambda^{\prime \prime}$ bestehen. Da aber dia in den eckigen Klammern stehenden Functionen von $\lambda, \lambda^{\prime}, \lambda^{\prime \prime}$ unabhängig sind, so ergiebt sich aus der Theorie der trigonometrischen Reihen, dass sie gleich Null sein müssen für alle zwischen $-\pi$ und $\pi$ liegenden Werthe von $u, u^{\prime}, u^{\prime \prime}$, $\mathrm{d}$. h. für alle Werthe, welche diese Variabeln während der Integration durchlaufen.

Jede der vorstehenden Gleichungen liefert also eine Differentialgleichung zwischen den einzelnen Functionen $\Omega$. Setzt man der Symmetrie wegen in der ersten $m^{i}=\mu_{3 i}$, in der zweiten $m^{i}=\mu_{3 i+1}$, in der dritten $m^{i}=\mu_{3 i+2}$, so dass man für $i=0,1, \ldots N$ hat

$$
\text { (13.) } \quad \mu_{3 i}=\mu_{3 i+1}=\mu_{3 i+2}=m^{i} \text {, }
$$

so treten diese Gleichungen sämmtlich in die nämliche Form; man erhält für

$$
\begin{gathered}
h, j=0,1, \ldots 3 N+2 \\
\text { (14.) } \quad \mu_{h} \frac{\partial^{2} \Omega^{h j}}{\partial t^{2}}+\sum_{g=11}^{g=3 N+2}(h g) \Omega^{g j}=0 .
\end{gathered}
$$

Auf diese Weise ist das ursprüngliche Problem auf die Integration des vorstehenden Systems gewöhnlicher Differentialgleichungen mit Rücksicht auf die in (11.) gestellten Anfangsbedingungen zurückgeführt. $\mathrm{Zu}$ dieser Integration sind indessen einige Vorbereitungen erforderlich.

VII.

Vor Allem ist es nothwendig, gewisse Eigenschaften der Coefficienten (hg) festzustellen.

Zunächst ergiebt sich aus ihrer Entstehung, dass sie nicht bloss von der Zeit $t$ unabhängig sind, sondern auch von der Stelle, welche der Punkt

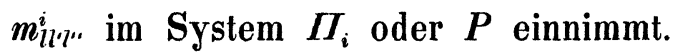

Eine zweite Eigenschaft ergiebt sich unmittelbar aus den Gleichungen (12.). Sie ist in der Gleichung

$$
(3 i+\chi, 3 k+\varrho)=(3 i+\varrho, 3 k+\chi)
$$

enthalten, wenn $\rho$ und $\chi$ nach Belieben aus den Zahlen 0, 1, 2 ausgewählt werden.

Die dritte Eigenschaft, deren Kenntniss nothwendig ist, besteht darin, dass die beiden Grössen ( $g h)$ und $(h g)$, welche im Allgemeinen complexe Werthe haben, stets zu einander conjugirt sind. 
Zum Beweise dieser Behauptung werden wir in den Ausdrücken der verschiedenen Grössen $(g h)$ die Vorzeichen der Indices $n, n^{\prime}, n^{\prime \prime}$ ändern, was auf den Werth dieser Ausdrücke offenbar keinen Einfluss hat.

Dann erkennt man zunächst leicht, dass die Grössen $(3 i+\chi, 3 i+\varrho)$ sämmtlich reell sind. Da ausserdem $(3 i+\chi, 3 i+\varrho)=(3 i+\varrho, 3 i+\chi)$ ist, so wird in diesem Falle der obigen Behauptung nicht widersprochen.

Wir wollen nun unter der Voraussetzung, dass $k$ von $i$ verschieden ist, die beiden Factoren untersuchen, welche im Ausdrucke von $(3 i+\chi, 3 k+\varrho)$ unter dem Summenzeichen stehen.

Da man nach art. I. und II.

$$
x_{k}^{\prime}-x_{i}=\alpha_{k}-\alpha_{i}+a n+a^{\prime} n^{\prime}+a^{\prime \prime} n^{\prime \prime}, \quad x_{i}^{\prime}-x_{k}=\alpha_{i}-\alpha_{k}+a n+\cdots
$$

hat, und ähnliche Gleichungen für die anderen Coordinatendifferenzen gelten, so folgt, dass $x_{k}^{\prime}-x_{i}, y_{k}^{\prime}-y_{i}, z_{k}^{\prime}-z_{i}$ durch Aenderung des Vorzeichens von $n, n^{\prime}, n^{\prime \prime}$ in $-\left(x_{i}^{\prime}-x_{k}\right),-\left(y_{i}^{\prime}-y_{k}\right),-\left(z_{i}^{\prime}-z_{k}\right)$ verwandelt werden. Gleichzeitig gehen also die Ausdrücke

$$
\frac{\partial^{2}[i k]}{\partial x_{k}^{\prime 2}}, \frac{\partial^{2}[i k]}{\partial y_{i}^{\prime} \partial z_{k}^{\prime}} \text { u. s. w. über in } \frac{\partial^{2}[k i]}{\partial x_{i}^{\prime 2}}, \frac{\partial^{2}[k i]}{\partial y_{i}^{\prime} \partial z_{i}^{\prime}} \text { u. s. } \mathbf{w} \text {. }
$$

Hieraus folgt, dass in den Gleichungen (12.) der Zeichenwechsel der Indices $n, n^{\prime}, n^{\prime \prime}$ nur zur Folge hat, dass auf der linken Seíte 1) die Zahlen $i$ und $k$ vertauscht werden, und 2) in der Exponentialgrösse $\omega=e^{-(n u+\ldots) v-1}$ die $V_{-1}$ das entgegengesetzte Zeichen erhält. Dadurch wird aber die Grösse $(3 i+\chi, 3 k+\varrho)$ ohne Aenderung ihres Werthes in die Conjugirte von $(3 k+\chi, 3 i+\varrho)=(3 k+\varrho, 3 i+\chi)$ verwandelt, w. z. b. w.

Man kann endlich noch eine vierte Eigenschaft dieser Grössen $(g h)$ bemerken; dieselben sind nämlich in Bezug auf jede der drei Variabeln $u, u^{\prime}, u^{\prime \prime}$ periodisch, mit der Periode $2 \pi$. Daraus zieht man leicht den Schluss, dass die durch die Gleichungen (11.) und (14.) bestimmten Functionen $\Omega$ die nämliche Periodicität darbieten müssen.

VIII.

Setzt man

$$
\psi^{j}=\sum_{g, h}(h g) \Omega^{g^{j}} H^{h}, \quad \varphi^{j}=\sum_{h} \mu_{h} \Omega^{h j} H^{h},
$$

so kann man die Gleichungen (14.) in folgende Form bringen:

$$
\text { (15.) } \frac{\partial^{2}}{\partial t^{2}}\left(\frac{\partial \varphi^{j}}{\partial H^{h}}\right)+\frac{\partial \psi^{j}}{\partial H^{h}}=0, \quad h=0,1, \ldots 3 N+2 \text {. }
$$


Da diese Gleichungen linear, und ihre Coefficienten constant sind, so hăngt ihre Integration, wie bekannt, nur von der Untersuchung der Determinante der bilinearen Function

$$
\psi^{j}-x \varphi^{j}
$$

und ihrer Unterdeterminanten ab.

Vergleicht man aber die Ausdrücke der Functionen $\psi^{j}$ und $\varphi^{j}$ mit denjenigen, welche in der Abhandlung über die Verallgemeinerung einiger Theoreme des Herrn Weierstrass (p. 255 dieses Bandes) durch $\psi$ und $\varphi$ bezeichnet wurden, so erkennt man sofort, dass die dort gemachten Voraussetzungen im gegenwärtigen Falle erfüllt sind.

Setzt man nämlich $[h h]=\mu_{h}$ und, so lange $g$ von $h$ verschieden ist, $[g h]=0$, so wird

$$
\varphi^{j}=\Sigma[h g] \Omega^{o j} H^{h},
$$

und es sind nun $(g h)$ und $(h g)$, sowie $[g h]$ und $[h g]$ zu einander conjugirt, wie dort verlangt wurde. Setzt man ferner $\Omega^{h j}=x_{h}+i y_{h}, H^{h}=x_{h}-i y_{h}$, so wird $\varphi^{j}=\sum_{\imath} u_{h}\left(x_{h}^{2}+y_{h}^{2}\right)$. Dies kann, da sämmtliche $"$ als Massen materieller Punkte positiv sind, nur verschwinden, wenn alle reellen Variabeln $x$ und $y$ sich zugleich auf Null reduciren.

Bezeichnet man daher durch

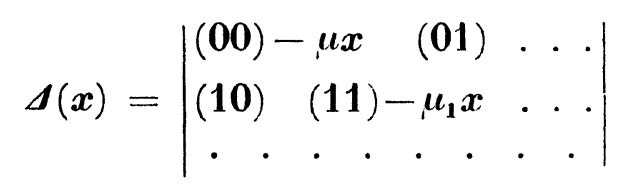

die Determinante von $\psi^{j}-x \varphi^{j}$, und durch $\Delta_{i k}(x)$ die aus einer Aenderung des Elementes (ik) entspringende Unterdeterminante von $\boldsymbol{S}(\boldsymbol{x})$, so folgt

I. dass alle Wurzeln der Gleichung $\mathscr{A}(\boldsymbol{x})=0$ reell sind.

Bezeichnet man die von einander verschiedenen Wurzeln dieser Gleichung $3 N+3^{\text {ten }}$ Grades durch

so folgt weiter,

$$
\boldsymbol{s}^{2}, \boldsymbol{s}_{1}^{2}, \ldots \boldsymbol{s}_{p}^{2},
$$

II. dass man durch Zerfällung in Partialbrüche

$$
\frac{\Delta_{i k}(x)}{\Delta(x)}=\sum_{\delta} \frac{A_{i k}^{\delta}}{s_{\delta}^{2}-x}
$$

erhält, wo die Factoren $\boldsymbol{A}$ von $x$ unabhängig sind, und die Summation sich über die verschiedenen Wurzeln der Gleichung $\Delta(x)=0$ erstreckt. 
Endlich. zieht man aus den Gleichungen (9.) der angeführten Abhandlung für den vorliegenden Fall die folgenden:

$$
\text { (III.) }\left\{\begin{array}{l}
\Omega^{g j}=\sum_{\delta} \sum_{h} A_{g h}^{\delta} \frac{\partial \varphi^{j}}{\partial \boldsymbol{H}^{h}}, \\
\boldsymbol{\Sigma}_{\boldsymbol{h}} \boldsymbol{A}_{\boldsymbol{g} h}^{\delta} \frac{\partial \boldsymbol{\psi}^{j}}{\partial \boldsymbol{H}^{h}}=\boldsymbol{s}_{\delta}^{2} \boldsymbol{\Sigma}_{\boldsymbol{h}} \boldsymbol{A}_{\boldsymbol{g} h}^{\delta} \frac{\partial \varphi^{j}}{\partial \boldsymbol{H}^{h}},
\end{array}\right.
$$

welche bei jeder Bedeutung der Grössen $\Omega^{{ }^{j} j}, \Omega^{1 j}, \ldots$ stattfinden.

Setzt man also

$$
\text { (16.) } \quad \sum_{h} A_{g h}^{\delta} \frac{\partial \varphi^{j}}{\partial H^{h}}=\sum_{h} \mu_{h} A_{g h}^{\delta} \Omega^{h j}=\Omega^{g j \delta},
$$

so folgt aus den Gleichungen (III.)

$$
\left\{\begin{aligned}
\Omega^{g j} & =\sum_{\delta} \Omega^{g j \delta} \\
\sum_{h} A_{g h}^{\delta} \frac{\partial \psi^{j}}{\partial H^{h}} & =s_{\delta}^{2} \Omega^{g j \delta}
\end{aligned}\right.
$$

Dies festgestellt, multipliciren wir die Gleichung (15.) mit $A_{g h}^{\delta}$, und addiren hierauf die aus ihr für $h=0,1, \ldots 3 N+2$ entspringenden Gleichungen zu einander; dann folgt

$$
\frac{\partial^{2} \Omega^{g j \delta}}{\partial t^{2}}+s_{\delta}^{2} \Omega^{g j \delta}=\mathbf{0} .
$$

Da man aus (16.) und (11.) für $t=0$

$$
\Omega^{g j \delta}=\mu_{j} A_{g j}^{\delta} \mathfrak{A}^{j}, \quad \frac{\partial \Omega^{g j \delta}}{\partial t}=\mu_{j} A_{g_{j}}^{\delta} \mathfrak{B}^{j}
$$

erhält, so ist also für ein beliebiges $t$

$$
\Omega^{g j \delta}=\mu_{j} A_{g j}^{\delta}\left[\mathfrak{U P}^{j} \cos s_{\delta} t+\mathfrak{B}^{j} \frac{\sin s_{\delta} t}{s_{\delta}}\right],
$$

mithin, wegen der Gleichung (17.)

$$
\Omega^{g j}=\mu_{j} \sum_{\delta} A_{g j}^{\delta}\left[\mathfrak{U}^{j} \cos s_{\delta} t+\mathfrak{B}^{j} \frac{\sin s_{\delta} t}{s_{\delta}}\right] .
$$

\section{IX.}

Aus dem eben gefundenen Ausdrucke der Functionen $\Omega$ ergiebt sich mit Rücksicht auf die Gleichung (10.), dass man, wenn

$$
\begin{gathered}
\frac{1}{(2 \pi)^{3}} \sum \int A_{j h}^{\delta} \frac{\sin s_{\delta} t}{s_{\delta}} e^{\left(\lambda u+\lambda^{\prime} u^{\prime}+\lambda^{\prime \prime} u^{\prime \prime}\right) v-1} \partial u \partial u^{\prime} \partial u^{\prime \prime}=T_{\lambda \lambda^{\prime} \cdot \lambda^{\prime \prime}}^{h j} \\
-\pi<u, u^{\prime}, u^{\prime \prime}<\pi
\end{gathered}
$$


gesetzt wird,

$$
P_{\lambda \lambda^{\prime} \lambda^{\prime \prime}}^{h j}=\mu_{j}\left[\mathfrak{U}^{j} \frac{\partial T_{\lambda \lambda^{\prime} \lambda^{\prime \prime}}^{h j}}{\partial t}+\mathfrak{B}^{j} T_{\lambda \lambda^{\prime} \lambda^{\prime \prime}}^{h j}\right]
$$

erhält. Da ferner (art. V, I und II) $P$ gleich $R$ oder $S$ ist, je nachdem man für jedes $j \mathfrak{A}^{j}=1, \mathfrak{B}^{j}=0$ oder $\mathfrak{A}^{j}=0, \mathfrak{B}^{j}=1$ setzt, so folgt:

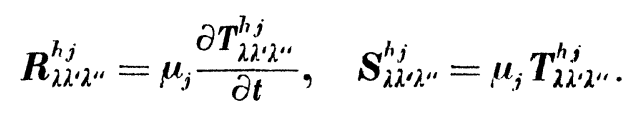

Durch Einsetzung dieser Ausdrücke erhält man aus den Gleichungen (9.) die Lösung des vorgelegten Problems. Sei zur besseren Uebersicht für $\varrho=0,1,2$

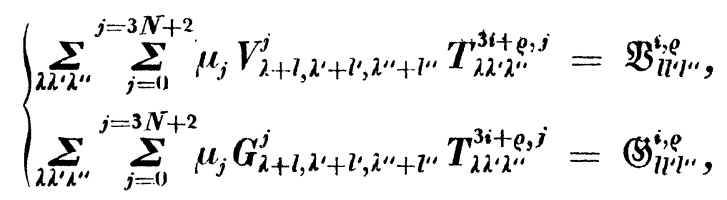

so folgt:

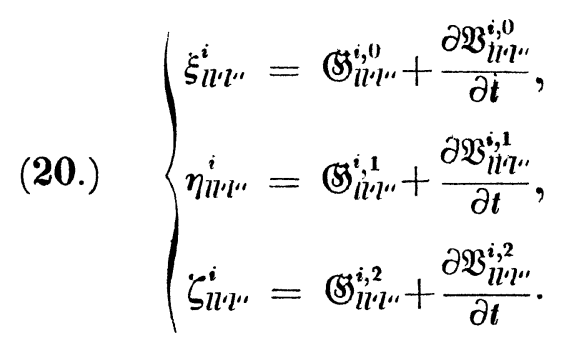

Die Einfachheit dieser Formeln lässt nichts zu wünschen übrig. Es scheint indessen schwer zu sein, aus ihnen unmittelbar eine Vorstellung von der durch sie dargestellten Bewegung zu gewinnen. Dagegen bieten sie für ạngenäherte Bestimmungen eine völlig zuverlässige Grundlage dar, bei welcher es nicht, wie bei der directen Behandlung der ursprünglichen Differentialgleichungen, zu befürchten ist, dass durch wiederholte Vernachlässigungen schliesslich alles verwischt wird, was den Charakter des ursprünglichen Problems ausmacht. 